\title{
The relation between differential leukocyte count, neutrophil to lymphocyte ratio and the presence and severity of coronary artery disease
}

\author{
Hatice Selcuk ${ }^{*}$, Lale Dinc, Mehmet Timur Selcuk, Orhan Maden, Ahmet Temizhan \\ Cardiology Department, Turkiye Yuksek Ihtisas Hospital, Ankara, Turkey \\ Email: ${ }^{*}$ haticsel@hotmail.com
}

Received 24 November 2011; revised 23 February 2012; accepted 16 May 2012

\begin{abstract}
Objectives: Total leukocyte count, a marker of inflammation has long been reported to be related with coronary artery disease (CAD). Recently, differenttial leukocyte count and elevated neutrophil to lymphocyte ratio $(\mathrm{N} / \mathrm{L})$ has been shown to indicate an increased long-term risk of mortality in patients with stable CAD, myocardial infarction and undergoing cardiovascular surgery. These studies offer incremental prognostic value to total leukocyte count. We sought to determine the relationship between the leukocyte subtypes and $\mathrm{N} / \mathrm{L}$ ratio and the presence and extent of CAD. Study design: The study consisted of 107 patients [70 (65.4\%) men, 37 (34.6\%) women, mean age $59.5 \pm 10.6$ years] referred to coronary angiography with suspected CAD. The subjects were classified into two groups according to the presence of significant CAD and the extent of CAD was determined by the Gensini method. Results: The lymphocyte count was found to be lower $(2031 \pm 741$ cells $/ \mu L$ vs $2392 \pm 611$ cells/ $\mu L, p: 0.010)$ and the $\mathrm{N} / \mathrm{L}$ ratio was found to be higher in patients with CAD $(2.86 \pm 1.57$ vs $2.04 \pm 1.01, p<0.001)$. In correlation analysis, the lymphocyte count and N/L ratio were significantly correlated with the presence and severity of CAD. Multivariate analysis identified $\mathrm{N} / \mathrm{L}$ as the only independent predictor of CAD after adjustment for traditional cardiovascular risk factors [odds ratio: $1.961,95 \%$ confidence interval (1.223, 3.143), p: 0.005]. Conclusion: Our findings reveal that $\mathrm{N} / \mathrm{L}$ ratio, a simple marker which can be derived from a routine complete blood count test was significantly and independently related to presence and severity of coronary atherosclerosis.
\end{abstract}

Keywords: Leukocyte; Neutrophil; Lymphocyte; Coronary Artery Disease; Neutrophil; Lymphocyte Ratio

${ }^{*}$ Corresponding author.

\section{INTRODUCTION}

Atherosclerosis has been increasingly recognized as a complex and multifactorial inflammatory disease rather being a simply process of lipid accumulation of the wall of the medium sized and large arteries. Inflammation plays a key role in the initiation, progression and complications of atherosclerosis by mediating every stage of the atheroma development. It has been hypothesized that injury of the vascular wall leads to an inflammatory response that involves complex interactions between endothelial and smooth muscle cells, leucocytes and plate-lets [1].

Several epidemiological and clinical studies reported associations between the various circulating markers of inflammation, such as C-reactive protein, fibrinogen, adhesion molecules, cytokines, elevated leukocyte count and the different clinical manifestations of coronary heart disease [2]. Elevated leukocyte count, a marker of inflammation, has long been identified as an independent predictor of an increased risk for long-term mortality and myocardial infarction both in individuals without cardiovascular disease at baseline and in patients with established coronary artery disease (CAD) [3-8]. Recently, the differential leukocyte count and elevated neutrophil to lymphocyte $(\mathrm{N} / \mathrm{L})$ ratio have been the subject of interest in predicting the risk for future cardiovascular events. An elevated N/L ratio has been shown to independently indicate an increased longterm risk of mortality in patients with stable CAD and those with myocardial infarction and offer incremental prognostic value to total leukocyte count [9-11]. However, the relation between the differential leukocyte count, N/L ratio and the presence and severity of CAD has not been extensively studied. In the current study, we sought to investigate the relationship between the leukocyte subtypes and the presence and severity of CAD assessed by Gensini method in patients with suspected CAD. 


\section{MATERIALS AND METHODS}

Our study prospectively evaluated 107 consecutive patients who underwent diagnostic coronary angiography in our clinic between May 2009 and June 2009.

All study patients referred to coronary angiography due to the angina or anginalike chest pain or positive noninvasive stress test result. Exclusion criteria were the presence of acute coronary syndromes, hematologic disorders, known CAD, infectious or inflammatory disease and severe renal or liver disease. Also the patients on statin therapy were excluded in order to prevent the confounding antiinflammatory effect of statins.

After detailed physical examination, the demographic characteristics and the cardiovascular risk factors were recorded by the physicians. Diabetes was defined as use of insulin or oral hypoglycemics if glucose levels exceeded $126 \mathrm{mg} / \mathrm{dl}$. Hyperlipidemia was defined as serum total cholesterol concentration $>200 \mathrm{mg} / \mathrm{dl}$, or serum LDL concentration $\geq 130 \mathrm{mg} / \mathrm{dl}$. The patients with a history of hypertension and who were taking antihy pertensive drugs were accepted as having hypertension. Body mass index (BMI) was calculated as the weight in kilograms divided by the square of the height in meters $\left(\mathrm{kg} / \mathrm{m}^{2}\right)$.

All patients and controls have given informed consent and the study protocol was approved by our institutional investigational review board.

\subsection{Laboratory Measurements}

Venous blood samples were collected before the day of coronary angiography for analysis of the following parameters using standard techniques: glucose, triglycerides, total cholesterol, high density lipoprotein (HDL) cholesterol, and low density lipoprotein (LDL) cholesterol. Total leukocyte count and differential leukocyte counts were measured with an automated Advia 2120 hematology analyzer (Roche).

\subsection{Coronary Angiography and Determination of Severity of CAD}

Coronary angiography was performed by the Judkins technique through femoral artery access. Coronary arteries were visualized in right and left oblique planes with cranial and caudal angulations. Evaluation of all coronary angiograms was made by two observers who were blinded to the clinical and laboratory data. Significant $\mathrm{CAD}$ on coronary angiograms was defined as stenosis of $50 \%$ or greater narrowing of the diameter in coronary arteries. Gensini score was used to give a measure of severity of coronary atherosclerosis. This method defines narrowing of the lumen of the coronary arteries as one for $1 \%-25 \%$ stenosis, two for $26 \%-50 \%$ stenosis, four for $51 \%-75 \%$ stenosis, eight for $76 \%-90 \%$ stenosis, and 32 for total occlusion. The score is then multiplied by a factor that represents the importance of the lesion's position in the coronary arterial system. For example, 5 for the left main coronary artery, 2.5 for the proximal left anterior descending or proximal left circumflex artery, 1.5 for the mid-region, and 1 for the distal left anterior descending or mid-distal region of the left circumflex artery.

\subsection{Statistical Analysis}

Statistical analyses were performed using SPSS 11.5 packet program (SPSS Inc., Chicago, Illinois, USA). Categorical variables were presented as frequency values and were compared by chisquare test. Continuous variables were expressed as mean values \pm standard deviation and were compared with Student's t-test or Mann Whitney U test. The variables that found to be significant in univariate analysis and the traditional cardiovascular risk factors as potential covariates were entered into a multivariate logistic regression model to evaluate the effect of these parameters on CAD. The relationship between the Gensini score and continuous variables was assessed by Spearman's correlation test.

\section{RESULTS}

The study group consisted of 107 consecutive patients [70 (65.4\%) men, 37 (34.6\%) women] with an average age of $59.5 \pm 10.6$ years. The patients were categorized into two groups according to presence or absence of significant CAD. Of the 107 patients 65 of them diagnosed as having CAD. The baseline demographic and clinical characteristics of the two groups are presented in Table 1. The groups were similar in terms of age, gender, BMI, systolic and diastolic blood pressure (all $p>0.05$ ). The prevalence of cardiovascular risk factors such as hypertension, hyperlipidemia, diabetes mellitus, smoking and family history did not differ between the groups (all $p>0.05$ ). In addition, there was no difference in the laboratory findings and the medications at admission between the groups (all $p>0.05$ ). When the groups compared with respect to total and differential leukocyte counts the lymphocyte count was found to be lower $(2031 \pm 741$ cells $/ \mu \mathrm{L}$ vs $2392 \pm 611$ cells $/ \mu \mathrm{L}, p: 0.010)$ and the $\mathrm{N} / \mathrm{L}$ ratio was found to be higher in patients with CAD $(2.86 \pm 1.57$ vs $2.04 \pm 1.01, p<0.001)$, whereas total leukocyte, neutrophil, monocyte, basophil, and eosinophil counts were not significantly different between the patients with and without CAD (all $p>0.05$ ).

In correlation analysis, a significant negative correlation was found between the lymphocyte count and Gensini (rho: $-0.457, p<0.001$ ). Also, the $\mathrm{N} / \mathrm{L}$ ratio was positively correlated with the Gensini score (rho: 0.314, $p$ : 0.011) (Figure 1).

Univariate analysis showed an association between CAD, lymphocyte count and N/L ratio. A multiple regression analysis including lymphocyte count and N/L ratio and traditional cardiovascular risk factors as 
Table 1. Demographic, clinical and biochemical characteristics of patients with and without coronary artery disease.

\begin{tabular}{|c|c|c|c|}
\hline Parameters & $\begin{array}{c}\text { Without CAD } \\
\text { n: } 42\end{array}$ & $\begin{array}{c}\text { With CAD } \\
\text { n: } 65\end{array}$ & $p$ Value \\
\hline Age (years) & $57.7 \pm 10.8$ & $60.7 \pm 10.5$ & 0.159 \\
\hline Male/Female n (\%) & $25(59.5 \%) / 17(40.5 \%)$ & $45(69.2 \%) / 20(30.8 \%)$ & 0.303 \\
\hline BMI $\left(\mathrm{kg} / \mathrm{m}^{2}\right)$ & $29.2 \pm 6.0$ & $29.1 \pm 5.3$ & 0.968 \\
\hline Systolic BP (mmhg) & $121.7 \pm 14.6$ & $126.8 \pm 16.6$ & 0.121 \\
\hline Diastolic BP (mmhg) & $70.2 \pm 10.5$ & $72.3 \pm 10.3$ & 0.252 \\
\hline \multicolumn{4}{|l|}{ Cardiac risk factors, n (\%) } \\
\hline Hypertension & $17(40.5 \%)$ & $34(52.3 \%)$ & 0.231 \\
\hline Hyperlipidemia & $22(52.4 \%)$ & $31(47.7 \%)$ & 0.636 \\
\hline Diabetes mellitus & $10(23.8 \%)$ & $16(24.6 \%)$ & 0.924 \\
\hline Cigarette smoking & $8(19.0 \%)$ & $18(27.7 \%)$ & 0.309 \\
\hline Family history & $13(31.0 \%)$ & $12(18.5 \%)$ & 0.136 \\
\hline \multicolumn{4}{|l|}{ Laboratory findings (mg/dl) } \\
\hline Urea & $33.5 \pm 16.5$ & $37.4 \pm 11.0$ & 0.102 \\
\hline Creatinine & $0.77 \pm 0.15$ & $0.81 \pm 0.24$ & 0.375 \\
\hline Fasting blood glucose & $111.1 \pm 36.1$ & $115.3 \pm 46.1$ & 0.548 \\
\hline Total cholesterol & $198.8 \pm 42.7$ & $190.2 \pm 43.4$ & 0.314 \\
\hline LDL-cholesterol & $104.5 \pm 46.8$ & $90.8 \pm 48.8$ & 0.151 \\
\hline HDL-cholesterol & $47.2 \pm 15.8$ & $45.9 \pm 16.5$ & 0.607 \\
\hline Triglycerides & $185.1 \pm 129.7$ & $155.0 \pm 93.2$ & 0.221 \\
\hline \multicolumn{4}{|l|}{ Medications at admission, n (\%) } \\
\hline Aspirin & $19(45.3 \%)$ & $33(50.8 \%)$ & 0.576 \\
\hline Beta-blocker & $15(35.7 \%)$ & $27(41.5 \%)$ & 0.547 \\
\hline ACEI/ARB & $8(19.0 \%)$ & $22(33.8 \%)$ & 0.096 \\
\hline $\mathrm{CCB}$ & $8(19.0 \%)$ & $12(18.5 \%)$ & 0.939 \\
\hline Hemoglobin (g/dl) & $14.2 \pm 1.8$ & $14.3 \pm 1.7$ & 0.743 \\
\hline $\mathrm{WBC}($ cells $/ \mu \mathrm{L})$ & $7645 \pm 1672$ & $8209 \pm 2092$ & 0.145 \\
\hline Neutrophil (cells/ $\mu \mathrm{L})$ & $4504 \pm 1508$ & $5102 \pm 1664$ & 0.063 \\
\hline Lymphocyte (cells $/ \mu \mathrm{L}$ ) & $2392 \pm 611$ & $2031 \pm 741$ & $0.010^{*}$ \\
\hline Monocyte (cells $/ \mu \mathrm{L})$ & $609.5 \pm 333.4$ & $629.2 \pm 360.9$ & 0.815 \\
\hline Basophil (cells $/ \mu \mathrm{L}$ ) & $38.1 \pm 126.8$ & $23.1 \pm 46.0$ & 0.989 \\
\hline Eosinophil (cells/ $\mu \mathrm{L})$ & $161.9 \pm 132.4$ & $178.5 \pm 182.4$ & 0.554 \\
\hline Neutrophil/Lymphocyte ratio & $2.04 \pm 1.01$ & $2.86 \pm 1.57$ & $<0.001^{*}$ \\
\hline
\end{tabular}

ACEI, angiotensin converting inhibitor; $\mathrm{ARB}$, angiotensin receptor blocker; $\mathrm{BMI}$, body mass index; $\mathrm{BP}$, blood pressure; $\mathrm{CAD}$, coronary artery disease; $\mathrm{CCB}$, calcium canal blocker; HDL, high density lipoprotein; LDL, low density lipoprotein; WBC, white blood cell; "Statistically significant. 


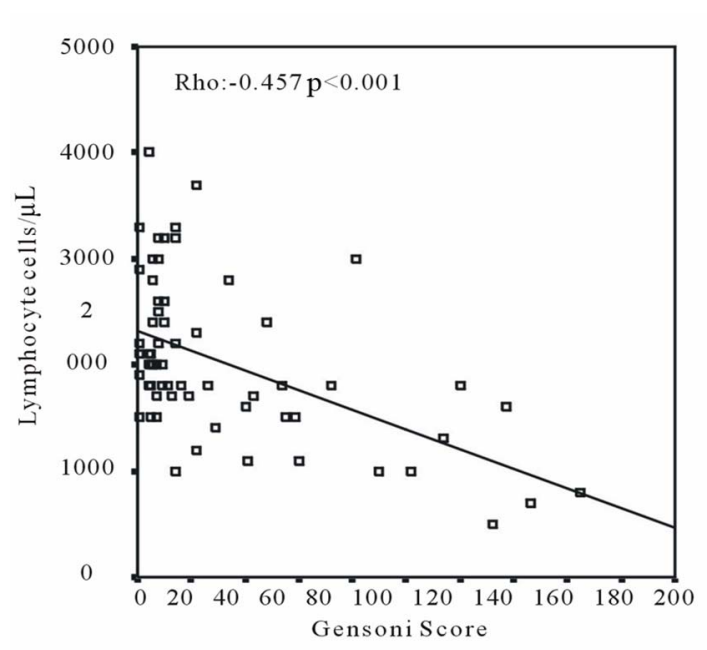

(a)

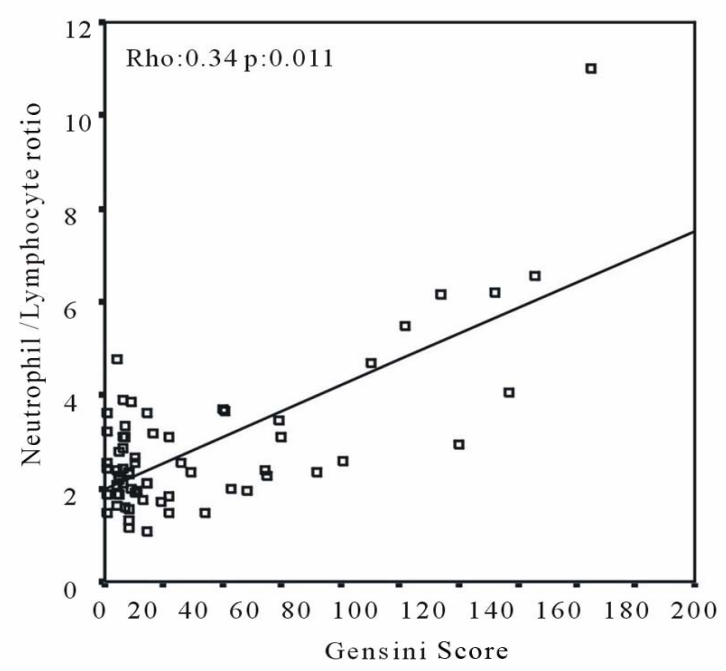

(b)

Figure 1. The relationship between lymphocyte count and Gensini score (a) and the relationship between neutrophil/lymphocyte ratio and Gensini score (b) in patients with coronary artery diseases.

potential covariates was performed in order to find their impact on CAD. Multivariate analysis identified N/L ratio as the only independent predictor for the presence of CAD [odds ratio: 1.961, 95\% confidental interval (1.223 - 3.143), $p$ : 0.005], whereas age, gender, diabetes mellitus, hypertension, hyperlipidemia, smoking, family history, total cholesterol, HDL cholesterol, LDL cholesterol, triglycerides and lymphocyte count were not associated with CAD (all $p>0.05)$.

\section{DISCUSSION}

The present study demonstrated that the lymphocyte co- unt is significantly decreased and the N/L ratio increased in patients with CAD. Both the lymphocyte count and the $\mathrm{N} / \mathrm{L}$ ratio correlated with the severity of CAD as assessed by Gensini score. In addition, multivariate analysis identified $\mathrm{N} / \mathrm{L}$ ratio as an independent predictor for CAD. Our findings provides an additional evidence for the important role for inflammation in the development of coronary atherosclerosis and suggest that the differential leukocyte count, an easily available, inexpensive and simple test is independently associated with CAD even in the context of established cardiovascular risk factors.

A number of previous epidemiologic and clinical studies have shown the clear association between the peripheral leukocyte count and the risk of adverse cardiovascular events either in patients with established CAD or free of disease at baseline. For instance, a retrospective analysis of the SOLVD trials showed that an elevated leukocyte count was related to an increased all- cause mortality risk in patients with left ventricular dysfunction compared to those with normal left ventricular function [3]. In the TACTICS TIMI18 Substudy, elevated leukocyte count was associated with impaired epicardial and myocardial perfusion, more extensive CAD and higher 6 month mortality, after adjustment for traditional risk factors and biomarkers [5]. A study in 6021 Japanese patients showed that an elevated leukocyte count may be a risk factor for acute coronary syndromes but not stable angina pectoris [4].

Several pathophysiologic mechanisms by which the leucocytes contribute to the pathogenesis of atherosclerosis have been proposed. One of the earliest steps in atherosclerotic process is the focal adherence of the circulating monocytes to the arterial intima following the arterial injury [2]. After engorged with lipid, monocytes differentiate into macrophages and secrete metalloproteinase enzymes [12,13]. Besides macrophages, neutrophils were shown to mediate endothelial cell detachment in vitro through digestion of endothelial cell surface proteins by secretion of proteolytic neutral proteases [14]. Neutrophils were shown to exhibit markedly increased chemotactic activity and leukotriene B4 in patients with stable angina [15]. Increased neutrophil aggregation and oxidase activity were demonstrated in the coronary sinuses of patients with angiographically documented CAD and stable angina [16]. Inflammatory cells not only play role in the initiation and progression of atherosclerosis but also take part in the plaque destabilization converting a chronic process into an acute ischemic event. Inflammatory processes leading to plaque rupture include the reduction of synthesis and degradation of the structural components of the fibrous cap by the matrix degrading enzymes and cytokines produced by macrophages at the site of the plaque rupture 
[13]. Although macrophages appear prominent in plaque disruption, neutrophil activation was proposed as one of the important features of an acute coronary event. Several in vitro and in vivo studies indicate the relevance of neutrophils in platelet activation, impairment of the coronary microcirculation, no reflow phenomenon and infarct extension [17-20]. Most of the investigations up to date suggest that neutrophils are the prominent subtype of the leukocytes participating particularly in acute coronary events.

Despite the excessive amount of evidence linking total leukocyte count to the $\mathrm{CAD}$, data regarding the contribution of specific subtypes of leucocytes to this relationship is limited. Up to date, relatively few studies examined the association between differential leukocyte counts and CAD in prospective follow up and in case control studies of different clinical forms of coronary heart disease and yielded conflicting results. Some studies reported independent relationships between the neutrophil counts whereas others with lymphocyte, eosinophil, monocyte counts or $\mathrm{N} / \mathrm{L}$ ratio and $\mathrm{CAD}$. In a large Japan cohort of individuals free of disease at baseline, elevated eosinophil, neutrophil and monocyte counts were found to be correlated with the incidence of CAD [21]. However, a high monocyte count was reported to be strongly associated with the risk of CAD in 3779 middleaged French men [22]. A study in patients with stable CAD, the total leucocytes and neutrophil counts were associated with the presence and severity of CAD, but the associations were not independent [23]. A metaanalysis of seven long-term studies, involving a total of 30,374 participants indicated that neutrophil count may be a stronger predictor of CAD risk than other leukocyte components [24]. However, it is important to note that, all of the cases involved in this analysis were either cardiac death and nonfatal myocardial infarctions apart from a few dozen cases of angina. The discrepant findings among these studies may be explained, at least in part, by the heterogenity of the inclusion criteria and the difference in the populations studied.

To the best of our knowledge, the present study is the first to examine the association between $\mathrm{N} / \mathrm{L}$ ratio and the presence and severity of CAD. A previous study demonstrated an association between the leukocyte and neutrophil count and the severity and extent of CAD assessed by Gensini score [25]. A study in Chinese patients with suspected or known CAD reported that the neutrophil count is independently associated with the angiographical characteristics of coronary atherosclerosis [26]. However, the patients were not investigated with respect to $\mathrm{N} / \mathrm{L}$ ratio in these reports. Our major finding that the elevated N/L ratio independently predicts the presence of CAD is in line with the results of previous follow-up studies investigating the prognostic value of
$\mathrm{N} / \mathrm{L}$ ratio in patients with CAD. Horne et al. Demonstrated that the greatest risk prediction in long-term is given by the N/L ratio in 3227 patients with either stable CAD or acute myocardial infarction when compared with total leukocytes or high neutrophil or low leukocyte counts alone. In addition, the authors concluded that the risk prediction achieved by the differential leukocyte count was similar to or greater than highsensitivity $\mathrm{C}$ reactive protein, a well established inflammatory marker [27]. Tamhane et al. demonstrated the N/L ratio as an independent predictor of in hospital and 6 month mortality in patients with acute coronary syndrome after adjusting for GRACE (Global Registry of Acute Coronary Events) score [9]. Nunez et al. identified N/L ratio as a predictor of long-term mortality in patients with ST segment elevation myocardial infarction, with a discriminative ability than total leukocyte count [10]. In a recent follow up study of angiographically stable CAD patients, $\mathrm{N} / \mathrm{L}$ ratio emerged an independent predictor of cardiac mortality [11]. The N/L ratio is thought to be a valuable marker for CAD than the neutrophil or lymphocyte count alone, as it combines the two different parameters that acts in opposite directions.

In the present study we found a negative correlation between the lymphocyte count and the severity of coronary atherosclerosis. Increasing evidence from experimental models of atherosclerosis revealed an important role of lymphocytes in regulating inflammatory response in atherosclerosis development [28]. The relative lymphocyte count has been previously shown to have prognostic utility in patients with CAD and congestive heart failure [29,30]. Recently, lymphopenia was reported to be associated with an increased risk for developing the combin ed end point of death or MI in patients with acute chest pain without enzymatic and electrocardiographic change [31]. It has been proposed that the activation of sympathetic nervous system in response to systemic stress causes an elevation in serum cortisol and the resultant shift in the leukocyte differential to a lower percentage of lymphocytes [32,33]. The correlation we found between the CAD and lymphopenia in the present study supports the notion that lymphopenia may be involved in the development of atherosclerosis.

The present study has some limitations including its relatively small sample size and crosssectional design. Due to the crosssectional design we cannot rule out the possibility of the effect of unmeasured potential confounders on the development and severity of CAD. In addition, because the CAD group included predominantly men, generalization of the results to women may be uncertain.

In conclusion, compared with subjects without significant $\mathrm{CAD}$, the patients with $\mathrm{CAD}$ had lower lymphocyte count and higher $\mathrm{N} / \mathrm{L}$ ratio and these variables were cor- 
related significantly with the presence and severity of CAD. Multivariate analysis identified N/L ratio as the sole independent predictor of CAD after adjusting for established cardiovascular risk factors. These findings suggest that inflammation plays an important role in coronary atherosclerosis and the addition of differential leukocyte count and N/L ratio measurement to the clinical evaluation, which can be determined by a routine complete blood count test may be useful in predicting the presence and severity of CAD.

\section{REFERENCES}

[1] Ross, R. (1999) Atherosclerosis-An inflammatory disease. The New England Journal of Medicine, 340, 115126. doi:10.1056/NEJM199901143400207

[2] Lind, L. (2003) Circulating markers of inflammation and atherosclerosis. Atherosclerosis, 169, 203-214. doi:10.1016/S0021-9150(03)00012-1

[3] Cooper, H.A., et al. (1999) White blood cell count and mortality in patients with ischemic and nonischemic left ventricular systolic dysfunction (an analysis of the studies of left ventricular dysfunction [solvd]). American Journal of Cardiology, 84, 252-257. doi:10.1016/S0002-9149(99)00272-6

[4] Takeda, Y., et al. (2003) Elevated white blood cell count as a risk factor of coronary artery disease: Inconsistency between forms of the disease. Japanese Heart Journal, 44, 201-211. doi:10.1536/jhj.44.201

[5] Sabatine, M.S., et al. (2002) Relationship between baseline white blood cell count and degree of coronary artery disease and mortality in patients with acute coronary syndromes: A tactics-timi 18 (treat angina with aggrastat and determine cost of therapy with an invasive or conservative strategy-thrombolysis in myocardial infarction 18 trial)substudy. Journal of the American College of Cardiology, 40, 1761-1768. doi:10.1016/S0735-1097(02)02484-1

[6] Phillips, A.N., et al. (1992) Leukocyte count and risk of major coronary heart disease events. American Journal of Epidemiology, 136, 59-70.

[7] Gillum, R.F., et al. (1993) White blood cell count, coronary heart disease, and death: The nhanes i epidemiologic follow-up study. American Heart Journal, 125, 855-863. doi:10.1016/0002-8703(93)90181-8

[8] Beydilli, I., et al. (2010) Importance of high sensitive c-reactive protein, leukocyte levels and neutrophyl/lymphocyte rate in the patients with acute coronary syndrome. Cardiovascular Sciences-Türkiye Klinikleri, 22, 61-68.

[9] Tamhane, U.U., et al. (2008) Association between admission neutrophil to lymphocyte ratio and outcomes in patients with acute coronary syndrome. American Journal of Cardiology, 102, 653-657. doi:10.1016/j.amjcard.2008.05.006

[10] Nunez, J., et al. (2008) Usefulness of the neutrophil to lymphocyte ratio in predicting long-term mortality in st segment elevation myocardial infarction. American Jour- nal of Cardiology, 101, 747-752. doi:10.1016/j.amjcard.2007.11.004

[11] Papa, A., et al. (2008) Predictive value of elevated neutrophil-lymphocyte ratio on cardiac mortality in patients with stable coronary artery disease. Clinica Chimica Acta, 395, 27-31. doi:10.1016/j.cca.2008.04.019

[12] Madjid, M., et al. (2004) Leukocyte count and coronary heart disease: Implications for risk assessment. Journal of the American College of Cardiology, 44, 1945-1956. doi:10.1016/j.jacc.2004.07.056

[13] Welt, F.G., et al. (2001) Atherosclerosis and plaque rupture. Catheterization and Cardiovascular Interventions, 53, 56-63. doi:10.1002/ccd.1130

[14] Harlan, J.M., et al. (1981) Neutrophil-mediated endothelial injury in vitro mechanisms of cell detachment. The Journal of Clinical Investigation, 68, 1394-1403. doi:10.1172/JCI110390

[15] Mehta, J., et al. (1989) Neutrophil function in ischemic heart disease. Circulation, 79, 549-556. doi:10.1161/01.CIR.79.3.549

[16] Ricevuti, G., et al. (1989) New trends in coronary artery disease: The role of granulocyte activation. Atherosclerosis, 78, 261-265. doi:10.1016/0021-9150(89)90232-3

[17] Lucchesi, B.R. (1990) Myocardial ischemia, reperfusion and free radical injury. American Journal of Cardiology, 65, 14I-23I. doi:10.1016/0002-9149(90)90120-P

[18] Mehta, J.L., et al. (1988) Neutrophils as potential participants in acute myocardial ischemia: Relevance to reperfusion. Journal of the American College of Cardiology, 11, 1309-1316. doi:10.1016/0735-1097(88)90297-5

[19] Jolly, S.R., et al. (1986) Reduction of myocardial infarct size by neutrophil depletion: Effect of duration of occlusion. American Heart Journal, 112, 682-690. doi:10.1016/0002-8703(86)90461-8

[20] Michelson, A.D., et al. (2001) Circulating monocyteplatelet aggregates are a more sensitive marker of in vivo platelet activation than platelet surface p-selectin: Studies in baboons, human coronary intervention, and human acute myocardial infarction. Circulation, 104, 1533-1537. doi:10.1161/hc3801.095588

[21] Prentice, R.L., et al. (1982) Leukocyte counts and coronary heart disease in a japanese cohort. American Journal of Epidemiology, 116, 496-509.

[22] Olivares, R., et al. (1993) Monocyte count: A risk factor for coronary heart disease? American Journal of Epidemiology, 137, 49-53.

[23] Rasouli, M., et al. (2007) Total and differential leukocytes counts, but not hscrp, esr, and five fractioned serum proteins have significant potency to predict stable coronary artery disease. Clinica Chimica Acta, 377, 127-132. doi:10.1016/j.cca.2006.09.009

[24] Wheeler, J.G., et al. (2004) Associations between differential leucocyte count and incident coronary heart disease: 1764 incident cases from seven prospective studies of 30,374 individuals. European Heart Journal, 25, $1287-$ 1292. doi:10.1016/j.ehj.2004.05.002

[25] Amaro, A., et al. (1993) Leukocyte count as a predictor 
of the severity ischaemic heart disease as evaluated by coronary angiography. Revista Portuguesa de Cardiologia, 12, 913-917.

[26] Jia, E.Z., et al. (2005) Relationship between leukocyte count and angiographical characteristics of coronary atherosclerosis. Acta Pharmacologica Sinica, 26, 10571062. doi:10.1111/j.1745-7254.2005.00169.x

[27] Horne, B.D., et al. (2005) Which white blood cell subtypes predict increased cardiovascular risk? Journal of the American College of Cardiology, 45, 1638-1643. doi:10.1016/j.jacc.2005.02.054

[28] Mallat, Z., et al. (2004) Immunomodulation to combat atherosclerosis: The potential role of immune regulatory cells. Expert Opinion on Biological Therapy, 4, 13871393. doi:10.1517/14712598.4.9.1387

[29] Ommen, S.R., et al. (1997) Usefulness of the lymphocyte concentration as a prognostic marker in coronary artery disease. American Journal of Cardiology, 79, 812-814. doi:10.1016/S0002-9149(96)00878-8
[30] Ommen, S.R., et al. (1998) Predictive power of the relative lymphocyte concentration in patients with advanced heart failure. Circulation, 97, 19-22. doi:10.1161/01.CIR.97.1.19

[31] Nunez, J., et al. (2009) Relationship between low lymphocyte count and major cardiac events in patients with acute chest pain, a non-diagnostic electrocardiogram and normal troponin levels. Atherosclerosis, 206, 251-257. doi:10.1016/j.atherosclerosis.2009.01.029

[32] Nelson, D.H., et al. (1952) Blood levels of 17-hydroxycorticosteroids following the administration of adrenal steroids and their relation to levels of circulating leukocytes. Journal of Clinical Investigation, 31, 843-849. doi:10.1172/JCI102671

[33] Thomson, S.P., et al. (1980) Endogenous cortisol: A regulator of the number of lymphocytes in peripheral blood. Clinical Immunology and Immunopathology, 17, 506514. doi:10.1016/0090-1229(80)90146-4 